\title{
Effects of retinal size on visual laterality
}

\author{
ANNETTE T. TAYLOR and JOSEPH B. HELLIGE \\ University of Southern California, Los Angeles, California
}

\begin{abstract}
Observers indicated whether two vertically presented bars were aligned one above the other (same trials) or were offset by an amount equal to the width of the bars on that trial (different trials). Retinal size was varied by using bars of three different widths (wide, medium, narrow). On different trials, reaction time to make correct responses was longer for narrow stimuli than for the other two sizes; this size effect was larger when stimuli were projected to the right visual field than when stimuli were projected to the left visual field. Such effects were not found on same trials. Implications of these results are discussed in light of alternative hypotheses about the effects of stimulus input parameters on visual laterality.
\end{abstract}

The present experiment examined the effect of retinal size on the processing of visual stimuli projected to the left visual field-right hemisphere (LVF-RH) and right visual field-left hemisphere (RVF-LH). The investigation of size is interesting because alternative hypotheses that have been advanced to account for the effects of other input parameters on visual laterality make different predictions about the manner in which size should interact with the visual field (hemisphere) to which a stimulus is presented.

Sergent (1982a, 1982b, 1983) explained the effects of many stimulus input parameters in terms of a difference between the left and right cerebral hemispheres in the efficient use of higher and lower ranges of visual spatial frequency, respectively. According to at least one version of this spatial-frequency theory, changes in input parameters that change the proportion of high and low spatial frequencies contained in the stimuli are predicted to change visual laterality effects (for discussions, see Michimata \& Hellige, in press; Sergent \& Hellige, 1986). Specifically, performance should be shifted in favor of the RVF-LH as stimuli are changed to contain proportionately higher spatial frequencies. It is the case that decreasing the retinal size of a stimulus increases the proportion of high- compared to low-frequency components (for discussion, see Michimata \& Hellige, in press). Thus, as retinal size is decreased, visual laterality should shift toward a RVF-LH advantage (cf. Sergent, 1982a, 1982b).

Many of the lateralized effects of stimulus input parameters are also consistent with the generalization that visual laterality shifts toward a LVF-RH advantage under conditions that increase the difficulty of the visuoperceptual stages of information processing. One way to manipulate the "perceptibility" of visual stimuli is to change their retinal size. As noted by Pring (1981), for

The research reported here was supported in part by National Science Foundation Grant BNS-8608893 to the second author. Requests for reprints should be sent to Joseph B. Hellige, Department of Psychology, University of Southern California, Los Angeles, CA 90089-1061. most tasks there is an optimum size, with performance deteriorating as stimuli become smaller or larger than the optimum. From this stimulus-perceptibility viewpoint, quite a different interaction is predicted between stimulus size and visual field. Specifically, any RVF-LH advantage is predicted to emerge for the stimulus size that is most easily processed (defined operationally as the size producing the best overall performance), with a shift toward a LVF-RH advantage as stimuli move away from the optimum size.

With these considerations in mind, we required the subjects in the present experiment to indicate as quickly as possible whether two vertically presented bars were aligned one above the other (same trials) or were offset by an amount equal to the width of the bars on that trial (different trials). This nonverbal task was chosen to make processing demands primarily on visuoperceptual stages of analysis, rather than on more cognitive stages. Retinal size was varied by using bars of three different widths. The widths were chosen on the basis of a pilot experiment that indicated that the narrowest stimuli would be processed more slowly than the other two sizes. According to the spatial-frequency theory outlined earlier, any RVF-LH advantage is predicted to be largest for the narrowest stimuli. In contrast, the stimulus-perceptibility hypothesis predicts that any RVF-LH advantage will be smallest for the narrowest stimuli.

\section{METHOD}

\section{Subjects}

The subjects were 16 male and 16 female volunteers from introductory psychology courses. All subjects were right-handed native speakers of English with normal or corrected-to-normal vision in both eyes.

\footnotetext{
Apparatus and Stimulus Materials

The subject was seated at a table facing a $44 \times 48 \mathrm{~cm}$ dark gray screen approximately $55 \mathrm{~cm}$ away. The screen was covered with black posterboard with two rectangular viewing windows cut out, one in each visual field, and a small circular opening cut midway between the two windows for presentation of a fixation dot. The subject's head was supported by a forehead stabilizer bar with a chinrest in order to help steady the gaze. In front of the subject on the tabletop was a $17 \times 35.5 \mathrm{~cm}$
} 
response console. On top of the console were two pairs of buttons; the innermost button of each pair was $7.5 \mathrm{~cm}$ from the center of the console and the two buttons within a pair were $4.0 \mathrm{~cm}$ apart. Above the two innermost buttons were two cards bearing the same label (same or different, counterbalanced across subjects), and above each of the two outermost buttons was a card with the opposite label. Stimulus items and the fixation dot were rear-projected at the appropriate times by a Gerbrands projection tachistoscope (Model G1176) controlled by a Gerbrands timer (Model 300-6T). An Apple II microprocessor recorded response accuracy and latency on each trial.

A white fixation dot spanning approximately $0.2^{\circ}$ of visual angle was prepared with a luminance of approximately $4.0 \mathrm{~cd} / \mathrm{m}^{2}$. Stimuli on each trial consisted of two vertical bars, which appeared on the screen as white bars with a luminance of approximately $4.0 \mathrm{~cd} / \mathrm{m}^{2}$. The two bars on a trial were positioned one directly above the other (same trials) or they were offset by an amount equal to the width of the bars on that trial (different trials). On half of the different trials the upper bar was closer than the lower bar to the center of the screen; on the remainder of the different trials the reverse was true. All of the bars were approximately $2.0^{\circ}$ of visual angle in height and the two bars of a pair were always separated by approximately $0.5^{\circ}$ of visual angle. Bars of three different widths were used, spanning approximately $0.5^{\circ}$ (narrow), $1.0^{\circ}$ (medium), and $2.0^{\circ}$ (wide) of visual angle. When projected on the screen, the edge of the bar pair nearest the center was approximately $4.5^{\circ}$ of visual angle from the center. Experimental trials consisted of 240 stimuli, containing 20 instances of each of 12 trial types defined by the orthogonal combination of bar width (narrow, medium, wide), visual field (LVF-RH, RVF-LH), and stimulus type (same, different). The order of presentation of these trial types was random, with the restriction that there be two instances of each type in successive sets of 24 trials.

\section{Procedure}

At the beginning of the experimental session, the subject was shown how to face the screen with his/her head positioned in the chinrest apparatus and how to place the index and middle fingers of each hand on the innermost and outermost response buttons, respectively. The subject was instructed to fixate his/her gaze on the fixation dot at the beginning of each trial and to maintain that fixation until after the stimuli had appeared and the subject had made a response. The nature of the task was explained and samples of same and different stimulus pairs were shown to the subject on a sheet of paper. The subject was told to press the two buttons labeled same if the two bars on a trial were positioned one directly above the other and to press the two buttons labeled different if the two bars on a trial were offset. The subject was told to respond as quickly as possible without making errors. Prior to the 240 experimental trials, each subject received 25 practice trials and was given a chance to ask questions about the procedure. Each trial began with the onset of the fixation dot for $1.0 \mathrm{sec}$. The offset of the fixation dot coincided with a 150-msec presentation of a bar pair. The intertrial interval was $5.0 \mathrm{sec}$, with an additional break averaging approximately $30 \mathrm{sec}$ between successive sets of 24 trials.

\section{RESULTS}

The percentage of errors and median reaction time (RT) of correct responses were computed for each of the 12 trial types defined by the orthogonal combination of bar width (narrow, medium, wide), visual field (LVF-RH, RVF-LH), and stimulus type (same, different). Figure 1 shows percentage of errors and RT for each of these 12 conditions. The error rates were too low to allow a meaningful statistical analysis. For RTs, an analysis of variance included the three variables noted above as within-subjects variables, with bar width broken into linear and quadratic trend components to allow a more precise examination of the effects of retinal size.

As shown in the lower panels of Figure 1, RT was faster to same stimuli than to different stimuli $[F(1,24)=4.59$,

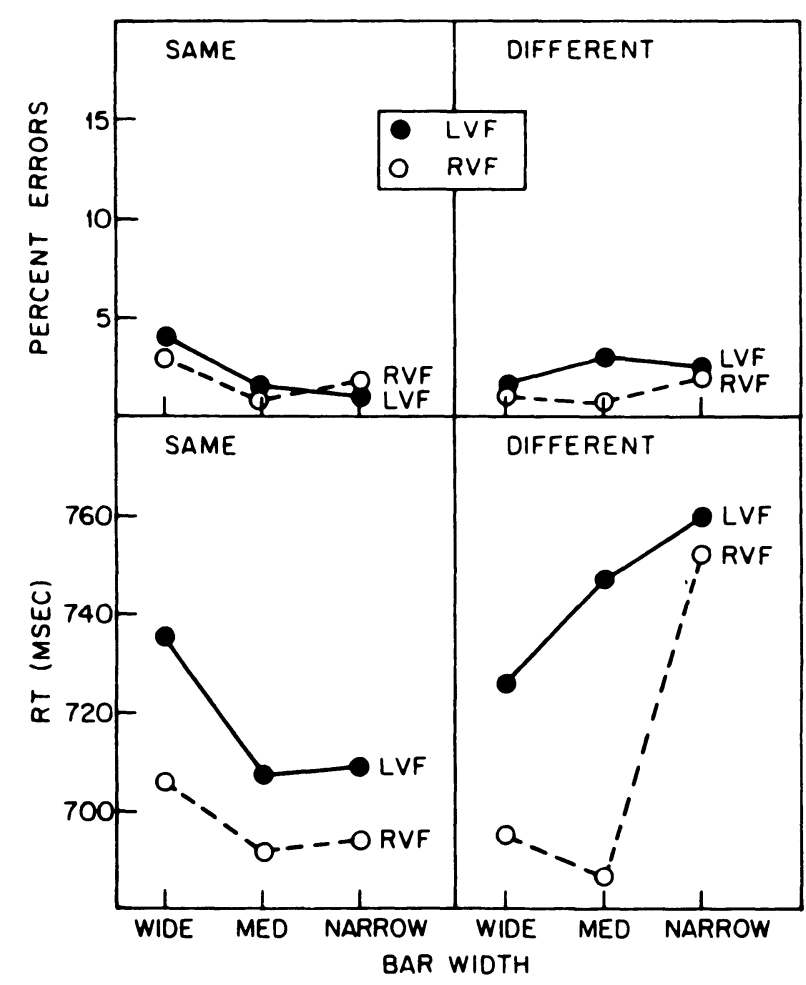

Figure 1. Percentage of errors (upper panels) and resction time (RT) of correct-responses (lower panch) as a function of stimulus width (wide, medium, and narrow). Results for same and different trials are shown in the left and right panels, respectively. The parameter in each panel is visual field of stimulus presentation.

$p<.05$ ] and faster on RVF-LH trials than on LVF-RH trials $[F(1,24)=12.16, p<.005]$. RT also differed with bar width: it was fastest to the medium bars, slowest to the narrow bars, and intermediate to the wide bars. As a result, there was a significant main effect of bar width $[F(2,48)=4.05, p<.05]$, with the main effect restricted to the quadratic component $[F(1,24)=6.62, p<.025]$. These main effects were qualified by several significant interactions. As can be seen in Figure 1, the bar-width main effect was restricted to different trials, producing a bar width $\times$ stimulus type interaction $[F(2,48)=10.31$, $p<.001$ ]. Of particular importance are interactions of bar width $\times$ visual field $[F(2,48)=3.09, p=.055]$ and bar width $\times$ visual field $\times$ stimulus type $[F(2,48)=3.64$, $p<.05$ ], with the latter interaction restricted to the quadratic component of bar width $[F(1,24)=7.45$, $p<.025]$. As shown in the bottom left panel of Figure 1, on same trials there was no bar width $X$ visual field interaction $(F<1)$. In contrast, on different trials (lower right panel of Figure 1), there was a highly significant quadratic bar width $\times$ visual field interaction $[F(1,24)=$ $10.90, p<.005]$. As Figure 1 shows, this occurs because the RVF-LH advantage is largest for medium-width stimuli and smallest for narrow-width stimuli. Subsequent analyses restricted to the wide and medium stimuli showed no main effect of bar width and no bar width $x$ visual field interaction. Subsequent analyses restricted to the 
medium and narrow stimuli showed both of these effects. Thus, both the bar-width main effect and the bar width $X$ visual field interaction noted earlier are accounted for by the shift from medium to narrow width.

\section{DISCUSSION}

Of the two possible explanations of stimulus input effects considered in the introduction, the present results favor the stimulus-perceptibility hypothesis. Specifically, when stimulus size became sufficiently smaller than the optimum size (i.e., was shifted from medium to narrow width), RT increased and did so significantly more on RVF-LH trials than on LVF-RH trials. A change in size that did not influence the overall performance level (wide to medium) did not produce a significant interaction with visual field.

The fact that both the main effect of bar width and the bar width $x$ visual field interaction were restricted to different trials was not anticipated, and the reasons for it are not clear. However, a similar finding was obtained by Michimata and Hellige (in press) for a task that required observers to indicate whether or not two nonlinguistic stimuli were physically identical. They reported that on trials on which the two stimuli were different from each other, large stimuli were processed more accurately and more rapidly than small stimuli, and this size effect was larger on RVF-LH trials than on LVF-RH trials. This is similar to the results on different trials in the present experiment. On trials on which the two stimuli were identical to each other, Michimata and Hellige reported that there was no main effect of size, and the interaction of size $\times$ visual field was quite different from that obtained when the stimuli were different. Specifically, on RVF-LH trials performance was better for small stimuli than for large stimuli, and on LVF-RH trials performance was better for large stimuli than for small stimuli-an interaction that is consistent with the predictions of spatial-frequency theory. It is interesting that both in a task that requires judgments of feature identity (Michimata \& Hellige, in press) and in a task that requires judgments of location identity (the present experiment), stimulus size influences performance only on different trials, and that the type of size $X$ visual field interaction predicted by the stimulus-perceptibility hypothesis is restricted to that condition in which there is evidence for a main effect of size. Further discussion of these same versus different effects must await studies designed explicitly to study them.

Although the present results are generally more consistent with an interpretation in terms of stimulus perceptibility, they do not provide unequivocal evidence against the notion that the two hemispheres differ in the efficient use of different ranges of spatial frequency. As noted elsewhere (e.g., Hellige \& Sergent, 1986; Sergent \& Hellige, 1986), stimuli of any size contain a wide range of spatial frequencies, so that manipulations of stimulus size only shift the predominance of various bandwidths, without necessarily eliminating the others. Although the results from different trials in both the present experiment and the experiments reported by Michimata and Hellige (in press) argue against a variation of the spatial-frequency theory in which the proportion of high and low frequencies is the relevant factor, other versions of the theory may be possible. For example, the absolute bandwidths required to perform the experimental task may be the determining factor. It could be argued that the present task requires subjects to note whether two edges (those of the top and bottom bars) are precisely aligned and that the frequencies that define the edges are independent of bar width. According to this view, a RVF-LH advantage could emerge if the left hemisphere is better able than the right to utilize the relevant frequencies, but there would be no bar width $x$ visual field interaction, as found on same trials. It is not clear, however, how this view could also accommodate the results of different trials, and to properly test such a a view it is necessary to specify in absolute terms the bandwidths that lead to most efficient processing in each cerebral hemisphere. Therefore, at the present time it is prudent to consider also the possibility that the effects of stimulus input parameters depend on variables in addition to visual spatial frequency.

\section{REFERENCES}

Hellige, J. B., \& Sergent, J. (1986). Role of task factors in visual field asymmetries. Brain \& Cognition, 5, 200-222.

Michimata, C., Hellige, J. B. (in press). Effects of blurring and stimulus size on the lateralized processing of nonverbal stimuli. Neuropsychologia.

PRING, T. R. (1981). The effects of stimulus size and exposure duration on visual field asymmetries. Cortex, 17, 227-240.

SERGENT, J. (1982a). The cerebral balance of power: Confrontation or cooperation? Journal of Experimental Psychology: Human Perception \& Performance, 8, 253-272.

SERGENT, J. (1982b). Methodological and theoretical consequences of variations in exposure duration in visual laterality studies. Perception \& Psychophysics, 31, 451-461.

SERGENT, J. (1983). The effects of sensory limitations on hemispheric processing. Canadian Journal of Psychology, 37, 345-366.

SERGENT, J., \& Hellige, J. B. (1986). Role of input factors in visualfield asymmetries. Brain \& Cognition, 5, 174-199.

(Manuscript received for publication March 10, 1987.) 